\title{
Banque mondiale : un séminaire en français "Choix stratégiques en matière de réformes éducatives »
}

\section{Christine Mousny}

\section{CpenEdition}

\section{Journals}

Édition électronique

URL : https://journals.openedition.org/ries/1812

DOI : $10.4000 /$ ries. 1812

ISSN : 2261-4265

Éditeur

France Education international

\section{Édition imprimée}

Date de publication : 1 septembre 2002

Pagination : 14-16

ISBN : 978-285420-554-1

ISSN : $1254-4590$

\section{Référence électronique}

Christine Mousny, «Banque mondiale : un séminaire en français « Choix stratégiques en matière de réformes éducatives » », Revue internationale d'éducation de Sèvres [En ligne], 30 | septembre 2002, mis en ligne le 24 novembre 2011, consulté le 06 juillet 2021. URL : http://journals.openedition.org/ries/ 1812 ; DOI : https://doi.org/10.4000/ries.1812

Ce document a été généré automatiquement le 6 juillet 2021

(c) Tous droits réservés 


\title{
Banque mondiale : un séminaire en français « Choix stratégiques en matière de réformes éducatives »
}

\author{
Christine Mousny
}

1 Le premier séminaire en français sur le thème des «choix stratégiques en matière de réformes éducatives ", organisé par l'Institut de la Banque mondiale et le Bureau de la Banque mondiale Moyen-Orient/Afrique du Nord (MENA), avec le concours du CIEP et de l'Institut international de planification de l'éducation (IIPE), s'est tenu au CIEP du 4 au 15 février 2002.

2 Depuis une quinzaine d'années, la Banque mondiale accompagne le développement du secteur de l'éducation dans les pays pauvres et à revenu intermédiaire. Convaincue du rôle essentiel que peuvent jouer l'éducation et la formation dans le développement des économies nationales, l'adaptation des ressources humaines aux besoins évolutifs du marché et le renforcement des démocraties, elle a fait des réformes dans ce secteur l'une de ses priorités et a développé son soutien au renforcement des capacités institutionnelles nécessaires à leur mise en œuvre par les pays.

3 La Banque a ainsi mis en place un important programme d'expertise, de conseil et de formation qui devrait permettre la diffusion à l'échelle mondiale de pratiques innovantes et de méthodes performantes. Un volet de ce programme, confié au Groupe Développement humain de l'Institut de la Banque mondiale, est spécialement consacré à la formation de tous ceux qui, dans les pays concernés, sont porteurs des réformes : décideurs politiques, hauts fonctionnaires, parlementaires, responsables syndicaux, représentants d'ONG, journalistes, membres des principales organisations de coopération. Les séminaires organisés jusqu’à présent s'étaient tenus à Washington en anglais.

4 Le séminaire francophone réalisé au CIEP a été conçu pour des responsables éducatifs des pays de la région MENA ${ }^{1}$ (Algérie, Maroc, Tunisie, Liban, Djibouti), ainsi que d'Afrique subsaharienne (Mauritanie, Côte d'Ivoire et Burkina Faso) dans la perspective de la préparation d'un séminaire ultérieur spécifique pour cette zone. La rencontre 
devait mettre l'accent sur l'amélioration de la qualité de l'éducation de base. Elle a été organisée autour de deux axes : conception et élaboration d'une réforme éducative, et amélioration de la qualité. Elle s'est appuyée sur des études de cas et des travaux de groupes, avec la présentation de diverses expériences nationales et de coopérations internationales.

5 Jean-Michel Sévérino ${ }^{2}$ a ouvert le séminaire par une conférence sur « L'éducation, bien public global ». En effet, la mondialisation, l'essor démographique et les progrès de la connaissance induisent des orientations nouvelles qui croisent inévitablement, en plein ou en creux, les objectifs de développement international adoptés par les Nations Unies, la Banque mondiale, le FMI et l'OCDE. C'est pourquoi l'accent a été mis d'emblée sur les nouvelles approches possibles en matière de stratégies de développement économique, de lutte contre la pauvreté et d'amélioration de l'éducation.

Les experts de l'Institut international de planification de l'éducation (IIPE) ont étudié, avec des ministres de l'éducation des pays invités, les éléments de la dynamique d'une réforme et les options stratégiques. Une place importante a été réservée aux fondements éthiques et culturels des systèmes éducatifs : dans une région où les identités religieuses et nationales occupent une place importante, il s'agit de véritables choix de société. Enfin, Mamadou N'Doye ${ }^{3}$ a insisté sur la prise en compte des risques et des freins dans la phase d'élaboration des réformes comme lors de leur mise en œuvre ainsi que sur la nécessité d'inclure dans la stratégie adoptée des marges de négociation, de concertation et de remédiation.

7 La deuxième partie du séminaire a été consacrée à la "qualité de l'éducation » entendue comme une notion non universelle et non généralisable. Des experts de l'IIPE ont proposé de la définir comme les « finalités définies par un système éducatif donné et les objectifs poursuivis qui posent la norme, à partir d'un état x de développement ». La réflexion a ensuite porté sur la l'évaluation d'un système éducatif (notamment à partir du dispositif français de la direction de la programmation et du développement du ministère de l'Éducation nationale), sur la relation pédagogique, la profession enseignante, l'accès et l'équité, ou encore sur le management de l'école, à partir d'exemples et d'expériences internationaux (France, Canada, Chili, Afrique du Sud).

La troisième partie du séminaire était réservée à des études de cas, à partir d'analyses et de témoignages de spécialistes français et étrangers. Les participants ont été accueillis à l'Institut universitaire de formation des maitres (IUFM), où leur a été présenté le dispositif français de formation des enseignants, sa spécificité et les raisons de sa mise en œuvre, ainsi qu'au Centre national d'enseignement à distance (CNED) et au Bureau européen de la Banque mondiale à Paris. Une vidéoconférence a été organisée avec Washington, Dakar et Santiago afin de recueillir les témoignages d'experts et de décideurs (ministre de l'Éducation chilien, secrétaire d'État à l'Enseignement supérieur du Brésil).

9 Outre son rôle logistique d'accueil des participants, le CIEP avait contribué à l'élaboration du programme du séminaire et au choix des intervenants. Il a également réalisé le classeur qui accompagne chaque formation de l'Institut de la Banque Mondiale et comporte, outre le résumé des interventions, une bibliographie et divers documents de référence (publications, revues, littérature grise, coupures de presse, etc.). Ce panorama en français des réflexions actuellement conduites sur les thèmes abordés durant le séminaire est consultable au centre de ressources documentaires du CIEP. 


\section{NOTES}

1. MENA : Moyen-Orient-Afrique du Nord.

2. Directeur général de l'Agence française de développement.

3. Secrétaire exécutif de l'ADEA et ancien ministre de l'Éducation du Sénégal.

\section{INDEX}

Mots-clés : politique éducative, réforme de l'enseignement, Banque mondiale, Banque de développement

\section{AUTEUR}

\section{CHRISTINE MOUSNY}

Chargée de programme au département Coopération en éducation du CIEP. 